\title{
Cantharidin Overcomes Imatinib Resistance by Depleting BCR-ABL in Chronic Myeloid Leukemia
}

\author{
Xiaoyan Sun ${ }^{1,2}$, Xueting Cai ${ }^{1,2}$, Jie Yang ${ }^{1,2}$, Jiao Chen ${ }^{1,2}$, Caixia Guo ${ }^{3, *}$, and Peng Cao ${ }^{1,2, *}$
}

\begin{abstract}
Cantharidin (CTD) is an active compound isolated from the traditional Chinese medicine blister beetle and displayed anticancer properties against various types of cancer cells. However, little is known about its effect on human chronic myeloid leukemia (CML) cells, including imatinib-resistant CML cells. The objective of this study was to investigate whether CTD could overcome imatinib resistance in imatinib-resistant CML cells and to explore the possible underlying mechanisms associated with the effect. Our results showed that CTD strongly inhibited the growth of both imatinib-sensitive and imatinib-resistant CML cells. CTD induced cell cycle arrest at mitotic phase and triggered DNA damage in CML cells. The ATM/ATR inhibitor CGK733 abrogated CTD-induced mitotic arrest but promoted the cytotoxic effects of CTD. In addition, we demonstrated that CTD downregulated the expression of the BCR-ABL protein and suppressed its downstream signal transduction. Real-time quantitative PCR revealed that CTD inhibited BCR-ABL at transcriptional level. Knockdown of BCR-ABL increased the cell-killing effects of CTD in K562 cells. These findings indicated that CTD overcomes imatinib resistance through depletion of BCRABL. Taken together, CTD is an important new candidate agent for CML therapy.
\end{abstract}

\section{INTRODUCTION}

Chronic myeloid leukemia (CML) is a myeloproliferative disorder caused by the constitutively active tyrosine kinase BCR$A B L$, the result of the reciprocal chromosomal translocation (9:22) (q34;q11) (Deininger et al., 2000; Pane et al., 1996). BCR-ABL abnormally activates multiple signal pathways, in-

\footnotetext{
${ }^{1}$ Affiliated Hospital of Integrated Traditional Chinese and Western Medicine, Nanjing University of Chinese Medicine, Nanjing 210028, China, 'Laboratory of Cellular and Molecular Biology, Jiangsu Province Academy of Traditional Chinese Medicine, Nanjing 210028, Jiangsu, China, ${ }^{3}$ Key Laboratory of Genomics and Precision Medicine, China Gastrointestinal Cancer Research Center, Beijing Institute of Genomics, Chinese Academy of Sciences, Beijing 100101, China

*Correspondence: guocx@big.ac.cn (CC); pcao79@yahoo.com (PC)
}

Received 25 January, 2016; revised 23 June, 2016; accepted 10 November, 2016; published online 13 December, 2016

Keywords: BCR-ABL, cantharidin, chronic myeloid leukemia, imatinib resistance cluding JAK-STAT, MAPK-ERK, and PI3K pathways, leading to deregulated proliferation and malignant transformation of $\mathrm{CML}$ cells (Danial and Rothman, 2000; Gesbert et al., 2000; Lugo et al., 1990). Targeting BCR-ABL with tyrosine kinase inhibitor (TKI), imatinib, has revolutionized the treatment of CML patients (Baselga and Arribas, 2004; Deininger et al., 2005). Unfortunately, about $20-30 \%$ of chronic phase CML patients are unresponsive to imatinib, owing to innate or acquired resistance, which has been a great challenge for CML therapy (Hochhaus et al., 2009). Although the second and third generations of tyrosine kinase inhibitors have been developed, their efficacy and safety in clinical use still need to be further investigated (Bixby and Talpaz, 2010). Therefore, exploring alternative treatment strategies is an urgent need.

Cantharidin (CTD) is a type of terpenoid extracted from the traditional Chinese medicine mylabris (blister beetle). A collective body of work from different groups has shown that CTD has strong anti-tumor effects in a wide range of tumor types, such as bladder cancer, colorectal cancer, hepatoma, pancreatic cancer, and breast cancer (Huang et al., 2011; Kuo et al., 2011; Li et al., 2010; 2011a; 2011b; Shou et al., 2013; Su et al., 2015; Zhang et al., 2014). It has been demonstrated that CTD blocks cell cycle at G2/M phase through multiple mechanisms. CTD induces G2/M cell cycle arrest by the JNK/Sp1 dependent downregulation of cyclin dependent kinase 1 and autophagydependent upregulation of p21 expression in human pancreatic cancer cells (Gong et al., 2015; Li et al., 2010). CTD also suppresses Cdc25c and cyclin A to trigger G2/M phase arrest in human melanoma cell line A375.S2 (Hsiao et al., 2014). In addition, CTD induces mitotic arrest, at least in part, by suppressing PP2Aa activity in A549 cells (Bonness et al., 2006).

The detailed mechanisms underlying the cell cycle arrest by CTD remain unclear. Furthermore, the therapeutic potential of CTD in imatinib-resistant CML cells has not been evaluated until now. Therefore, the aim of this study was to investigate whether CTD could overcome imatinib resistance in human CML cells and to explore the mechanisms underlying this activity.

\section{MATERIALS AND METHODS}

Reagents and antibodies

CTD (98\% or higher purity) was purchased from Shanghai Shifeng Biological Technology Company (China). CTD was dissolved in DMSO as a $40 \mathrm{mM}$ stock solution and stored at $20^{\circ} \mathrm{C}$. Primary antibodies against pCdc2, Cdc2, Cdc25c, cyclin B1, cyclin D1, pSTAT5, STAT5, pAKT, AKT, pERK1/2, 
ERK1/2, PARP1, cPARP, and pH3 (Ser10) were purchased from Cell Signal Technology, Inc. (USA). Antibodies against GAPDH and BCR were purchased from Santa Cruz Biotechnology (USA). Antibody against $\mathrm{YH} 2 \mathrm{AX}$ was from EMD Millipore (USA). Imatinib and CGK733 were purchased from Selleck (USA). Caffeine was purchased from Amquar Biology (AMQUAR Bio., USA). RPMI 1640 medium, fetal bovine serum (FBS), penicillin $G$ and streptomycin were provided by Gibco (USA). All the other reagents used in this study were of analytical grade.

\section{Cells and cell culture}

Human CML cell line K562 was purchased from Cell Bank of Shanghai Institute of Biochemistry and Cell Biology. K562 cells were cultured in RPMI 1640 medium supplemented with 10\% FBS. The imatinib-resistant cell line, K562R, was kindly provided by Guangbiao Zhou (Institute of Zoology, Chinese Academy of Sciences, China). K562R cells were cultured in RPMI 1640 medium supplemented with $10 \% \mathrm{FBS}$, and $1 \mu \mathrm{M}$ imatinib, which was removed before experiments with a wash-out period of 2 to 3 days.

Isolation of peripheral blood mononuclear cells (PBMCs) Normal blood PBMCs were isolated from healthy donors by density gradient centrifugation by Ficoll paque plus (GE Healthcare Life Sciences, Marlborough, USA) density sedimentation, followed by two washes in $1 \mathrm{x}$ phosphate buffered saline. Cells were then cultured in liquid culture (RPMI1640, supplemented with $20 \%$ FBS). Use of the PBMC samples was approved by the Institutional Review Board of Committee of Jiangsu Province Academy of Traditional Chinese Medicine.

\section{Cell proliferation and cell death}

Cells were seeded into a 96-well plate at a density of $1 \times 10^{4}$ cells/well, pre-cultured for $24 \mathrm{~h}$, and then treated with CTD at various concentrations $(0,5,10,20,40$, or $80 \mu \mathrm{M})$ for 24 or 48 h. Cell Counting Kit-8 (CCK-8) (Dojindo, Japan) was used to evaluate cell proliferation. Briefly, the medium from each well was removed after CTD treatment and $100 \mu$ of fresh serumfree medium with $10 \mu \mathrm{l}$ of CCK-8 was added. The absorbance was measured at $450 \mathrm{~nm}$ after further incubation for $2 \mathrm{~h}$ at $37^{\circ} \mathrm{C}$. Cell death was assessed by trypan blue dye exclusion test. After CTD treatment, cells were incubated with $0.4 \%$ trypan blue solution diluted with PBS. Stained cells and unstained cells were counted in a Neubauer chamber under microscope.

\section{Western blot}

Cells were collected and lysed with RIPA buffer containing protease inhibitor cocktail. The lysates were centrifuged and the supernatant was collected. Total proteins in the cells were quantitated by BCA protein assay, separated by sodium dodecyl sulfate polyacrylamide gel electrophoresis, and subsequently transferred onto a PVDF membrane. The membrane was blocked with $5 \%$ skimmed milk in TBS-T for $1 \mathrm{~h}$ at room temperature, then incubated with primary antibodies at $4^{\circ} \mathrm{C}$ overnight, followed by incubation with IRDye conjugated secondary antibody. All the antibodies were diluted in 5\% skimmed milk with TBS-T. The primary antibodies were diluted 1:500 $1: 1000$ and the secondary antibodies were diluted 1:5000. Odyssey infrared fluorescent scanner (LI-COR) was used for detecting the relevant proteins.

Hoechst 33258 staining

The cells were exposed to CTD at indicated concentrations for
$24 \mathrm{~h}$ and plated on glass slides by centrifuging using a cytospin. The Hoechst 33258 staining was done using the Hoechst staining kit (Beyotime, China). Briefly, cells were fixed with fixing buffer for 20 min and stained with Hoechst 33258 staining buffer for $15 \mathrm{~min}$. The cells were then observed under a confocal laser scanning microscope, Fluoview FV10i (Olympus) and analyzed using FV10-ASW4.0 software.

\section{G2/M cell cycle analysis}

The cell cycle was analyzed using FlowCellect ${ }^{\mathrm{TM}}$ bivariate cell cycle kit (EMD Millipore). CTD-treated cells were harvested, fixed, and permeabilized according to the instructions of the kit. The permeabilized cells were stained with anti-p-Histone H3AlexaFluor ${ }^{\circledR} 488$ antibody and propidium iodide/RNase solution. Fluorescence was analyzed using FACScan laser flow cytometry (Guava easyCyte HT, Millipore).

\section{yH2AX immunofluorescence staining}

Cells were plated on glass slides by centrifugation, fixed with $4 \%$ paraformaldehyde for $20 \mathrm{~min}$ and washed thrice with PBS. After permeabilizing with $0.3 \%$ Triton X-100 for $15 \mathrm{~min}$, the cells were blocked with $5 \%$ bovine serum albumin and incubated with antibody against $\mathrm{\gamma H} 2 \mathrm{AX}$ (diluted 1:1000) overnight at $4^{\circ} \mathrm{C}$, followed by incubation with Alexa Fluor 488 conjugated secondary antibody (diluted 1:1000). Nuclei were stained with DAPI. Fluorescence was observed with confocal laser scanning microscope Fluoview FV10i (Olympus) and analyzed using FV10-ASW4.0 software.

\section{RNA interference}

BCR-ABL specific siRNA and scramble RNA duplexes were transfected into K562 cells using lipofectamine 2000 (Invitrogen). The siRNA sequence for BCR-ABL was as follows: $5^{\prime}-$ GCAGAGUUCAAAAGCCCTT-3'. The scramble siRNA (negative control, NC) sequence was 5'-UUCUCCGAACGUGUCAC GUTT-3'. The knockdown efficiency was assessed by western blotting. Cell viability of BCR-ABL knockdown cells after treating with CTD was measured using CCK-8 assay.

\section{Real-time quantitative PCR (qRT-PCR)}

Total RNA was extracted from K562 and K562R cells using TRIzol reagent (Invitrogen). Five micrograms of total RNA was reverse transcribed using RNA PCR first-strand SuperScript Kit (Invitrogen). Fifty nanograms of total cDNA was used for qRTPCR with the SYBR Premix Ex Taq Kit (TaKaRa). Each PCR reaction was carried out in a total volume of $20 \mu \mathrm{l}$ on a 96-well optical reaction plate in Applied Bioscience 7500. The specific primers for qRT-PCR were BCR-ABL-sense: 5'-CATTCCGCT GACCATCAATAAG-3', BCR-ABL-antisense: 5'-GATGCTACT GGCCGCTGAAG-3', 18S-sense: 5'-AAACGGCTACCACATC CAAG-3', and 18S-antisense: 5'-CCTCCAATGGATCCTCGT TA-3'. The relative gene expression was normalized against 18S RNA expression.

\section{Statistics}

All experiments were performed at least three times and data were presented as the mean \pm SD. GraphPad Prism5.0 software (GraphPad Software Inc., USA) was used for statistical analyses. One-way analysis of variance (ANOVA) followed by post-hoc Dunnett's test was used to compare the treatment groups and the non-treatment group. The intensity of the immune-reactive bands in western blots was quantified by ImageJ software (National Institutes of Health). $P$ value less than 0.05 was considered as statistically significant. 
A

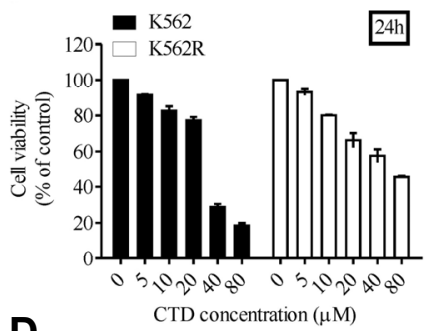

D

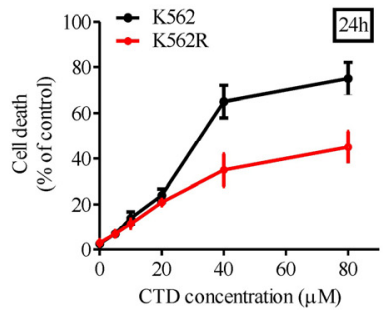

B

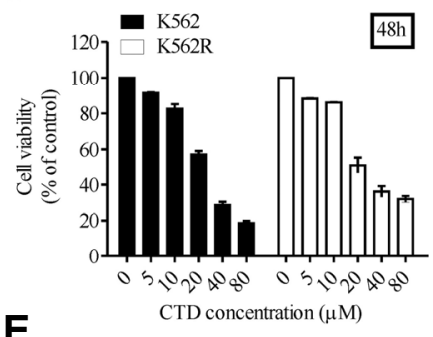

E

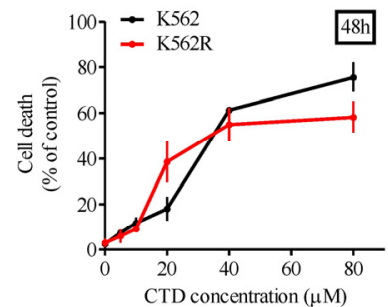

C

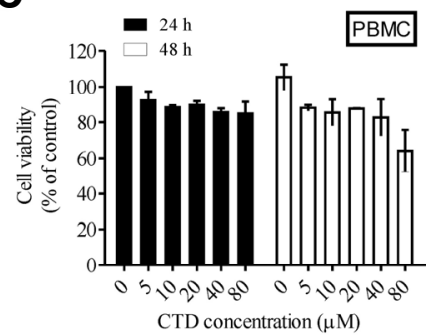

CTD concentration $(\mu \mathrm{M})$

Fig. 1. CTD inhibited the growth of CML cells. (A) Human CML cells $K 562$ and K562R were treated with indicated concentrations of CTD for $24 \mathrm{~h}$. Cell viability was measured using CCK-8 assay. (B) K562 and K562R cells were treated with indicated concentrations of CTD for $48 \mathrm{~h}$. Cell viability was evaluated by CCK-8 assay. (C) Normal human PBMCs were treated with indicated concentrations of CTD for 24 or $48 \mathrm{~h}$. Cell viability was measured by CCK-8 assay. (D) K562 and K562R cells were treated with indicated concentrations of CTD for $24 \mathrm{~h}$. Cell death was assessed by trypan blue dye exclusion assay. (E) K562 and K562R cells were treated with indicated concentrations of CTD for 48 h. Cell death was assessed by trypan blue dye exclusion assay. Data presented as the mean \pm SD of three independent experiments.

\section{RESULTS}

\section{CTD inhibited both imatinib-sensitive and}

imatinib-resistant CML cells

In this experiment, the imatinib-resistant CML cell line $\mathrm{K} 562 \mathrm{R}$ was used. We first characterized the resistance of K562R. Both K562 and K562R cells were treated with BCR-ABL kinase inhibitor, imatinib, at a concentration of $1 \mu \mathrm{M}$ for $48 \mathrm{~h}$. Apoptosis assay showed that $\mathrm{K} 562 \mathrm{R}$ cells exhibited strong resistance against imatinib-induced apoptosis compared with K562 cells (Supplementary Fig. S1A). Immunoblotting analysis showed that the protein levels of BCR-ABL did not change significantly in any of these cells (Supplementary Fig. S1B). STAT5 and ERK $1 / 2$ are downstream target proteins that are phosphorylated and activated by the tyrosine kinase, BCR-ABL. As shown in Supplementary Fig. S1B, imatinib treatment remarkably reduced the phosphorylation of STAT5 and ERK1/2 in K562 cells, whereas, the changes in K562R cells were insignificant. These results suggested that $\mathrm{K} 562 \mathrm{R}$ cells were resistant to imatinibinduced apoptosis and BCR-ABL downstream signaling pathway inhibition.

To investigate the anticancer potential of CTD against CML, the cytotoxicity of CTD toward normal PBMCs, imatinibsensitive CML cell line, K562, and imatinib-resistant cell line, K562R, was tested using CCK-8 assay. The results demonstrated that CTD suppressed the viability of both CML cell types (Figs. 1A and 1B) with little effect on normal blood cells (Fig. $1 \mathrm{C})$. The IC50 value of CTD for PBMCs $(>100 \mu \mathrm{M})$ was significantly higher than that for $\mathrm{K} 562$ and $\mathrm{K} 562 \mathrm{R}$ cells (28.23 and $54.42 \mu \mathrm{M}$, respectively) at $24 \mathrm{~h}$. The IC50 values for PBMCs, $\mathrm{K} 562$, and K562R cells at $48 \mathrm{~h}$ were 102.69, 27.63 and 31.34 $\mu \mathrm{M}$, respectively. Trypan blue exclusion assay showed that treatment of CTD induced cell death in K562 and K562R cells at the concentration of 5 to $80 \mu \mathrm{M}$ (Figs. 1D and 1E).
CTD induced mitotic arrest in CML cells

Morphologic changes of the cells were examined under phase contrast microscope. The normal spherical shape of K562 and K562R cells changed into unusual ellipsoid or spindle shape, with significant enlargement, after exposure to CTD (5-20 $\mu \mathrm{M})$ for $24 \mathrm{~h}$ (Fig. 2A). This result suggests that CTD treatment may result in a failure of cytokinesis in CML cells. The cell cycle can be divided into two distinct stages: the interphase stage and mitotic stage. In the second stage, or M-phase, chromatin condenses and cell division takes place. Previous studies have shown that Histone $\mathrm{H} 3$ phosphorylated $(\mathrm{pH} 3)$ at Ser10 could be a reliable and specific mitotic marker (Crosio et al., 2002). To examine whether CTD could trigger mitotic arrest in CML cells, we analyzed CTD-treated cells by flow cytometry after anti-pHistone H3/propidium iodide double staining. The results showed that CTD-treatment induced a significant increase in mitotic phase inK562 and K562R cells (Fig. 2B). As shown in Fig. 2C, after $24 \mathrm{~h}$ of CTD treatment, 19.2 to $24.5 \%$ of $\mathrm{K} 562$ cells were in mitotic phase, compared to only $1.6 \%$ of the control cells in mitotic phase; and 10.8 to $13.0 \%$ of K562R cells were in mitotic phase, compared to $3.11 \%$ of the control cells in mitotic phase. These results indicate that CTD induced mitotic failure in CML cells.

\section{Effects of CTD on cell cycle regulating proteins}

To further verify that CTD induced mitotic perturbation, we studied the changes in nuclear morphology after exposure to CTD. The cells underwent pronounced changes in nuclear morphology, including chromatin condensation (Fig. 3A). K562 cells with the abnormal mitotic nuclei accounted for about $1.05 \%$, $17 \%, 24 \%$ and $36 \%$ after treatment with CTD at the concentration of $0 \mu \mathrm{M}, 5 \mu \mathrm{M}, 10 \mu \mathrm{M}$, and $20 \mu \mathrm{M}$, respectively (Fig. 3B).

We next investigated the mechanism of CTD triggered mitotic arrest. Activation of cyclin B1/Cdc2 complex, a heterodimer 

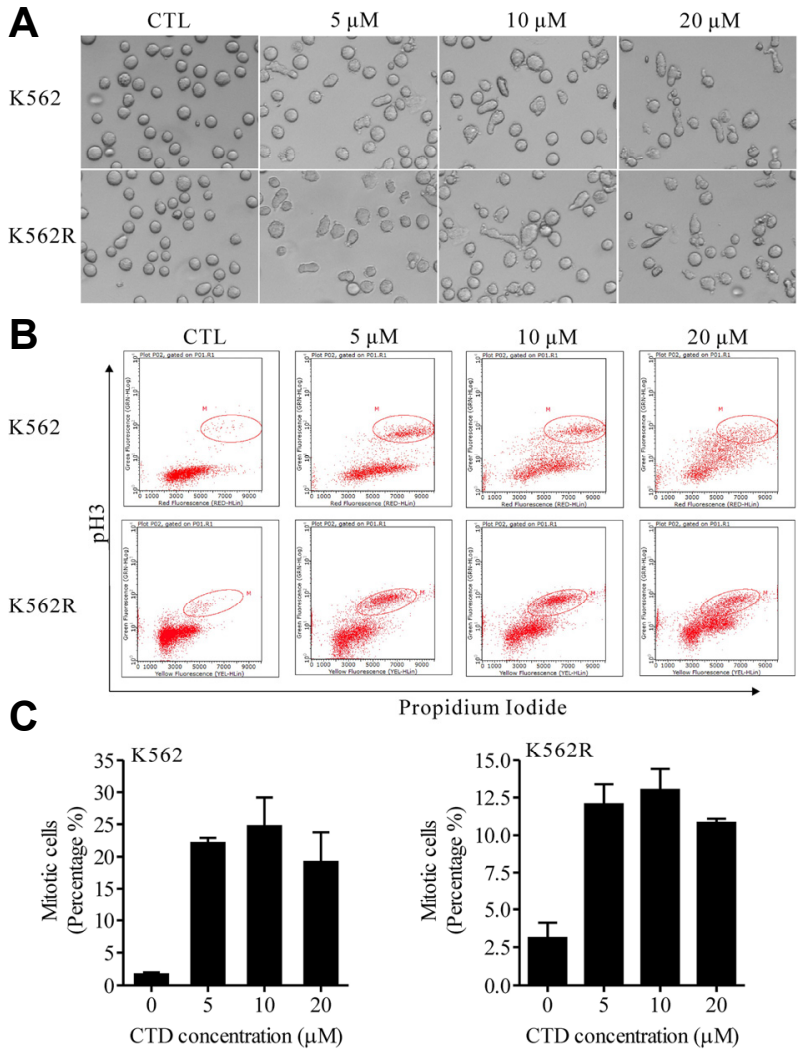

Fig. 2. CTD induced mitotic arrest in CML cells. (A) K562 and $\mathrm{K} 562 \mathrm{R}$ cells were treated with indicated concentrations of CTD for $24 \mathrm{~h}$, and the morphological changes were observed via microscopy. (B) K562 and K562R cells were incubated with indicated concentrations of CTD for $24 \mathrm{~h}$, and then stained with Anti-phosphoHistone H3 (Ser10) Alexa Fluor 488 /propidium iodide, and analyzed by flow cytometry. (C) Mitotic cells as a percentage of the total cells in each group. Data presented as the mean \pm SD of three independent experiments.

called mitosis-promoting factor, is essential for the transition of G2 to M phase. Upregulation of cyclin B1 is a typical marker of mitotic abnormality (Sánchez and Dynlacht, 2005; Wolanin et al., 2006). Therefore, we examined the expression levels of cyclin B1 by immunoblotting. As shown in Figs. 3C and 3D, CTD treatment caused a marked increase in the expression of cyclin B1 in K562 and K562R cells, respectively. Since dephosphorylation at Thr14 and Tyr15 of Cdc2 is crucial for its activation (Norbury et al., 1991), we next tested the phosphorylation status of Tyr 15 of Cdc2 in CTD-treated CML cells. The results showed that CTD reduced Tyr15-phosphorylated Cdc2 with no effect on the total protein level. Phosphatase, Cdc25c, is an upstream activator of Cdc2 through dephosphorylation at both Thr14 and Tyr15 sites (Gautier et al., 1991). We, therefore, examined the expression of Cdc25c and found a band shift of Cdc25c in a dose dependent manner. We also assessed the expression of cyclin D1, which drives the $\mathrm{G} 1$ to $\mathrm{S}$ phase transition and gets degraded in G2/M phase. The results showed the CTD-induced cyclin D1 reduction in both K562 (Fig. 3E) and K562R (Fig. 3F) cells. Taken together, these results demonstrated that CTD caused changes in mitotic signaling pathway.
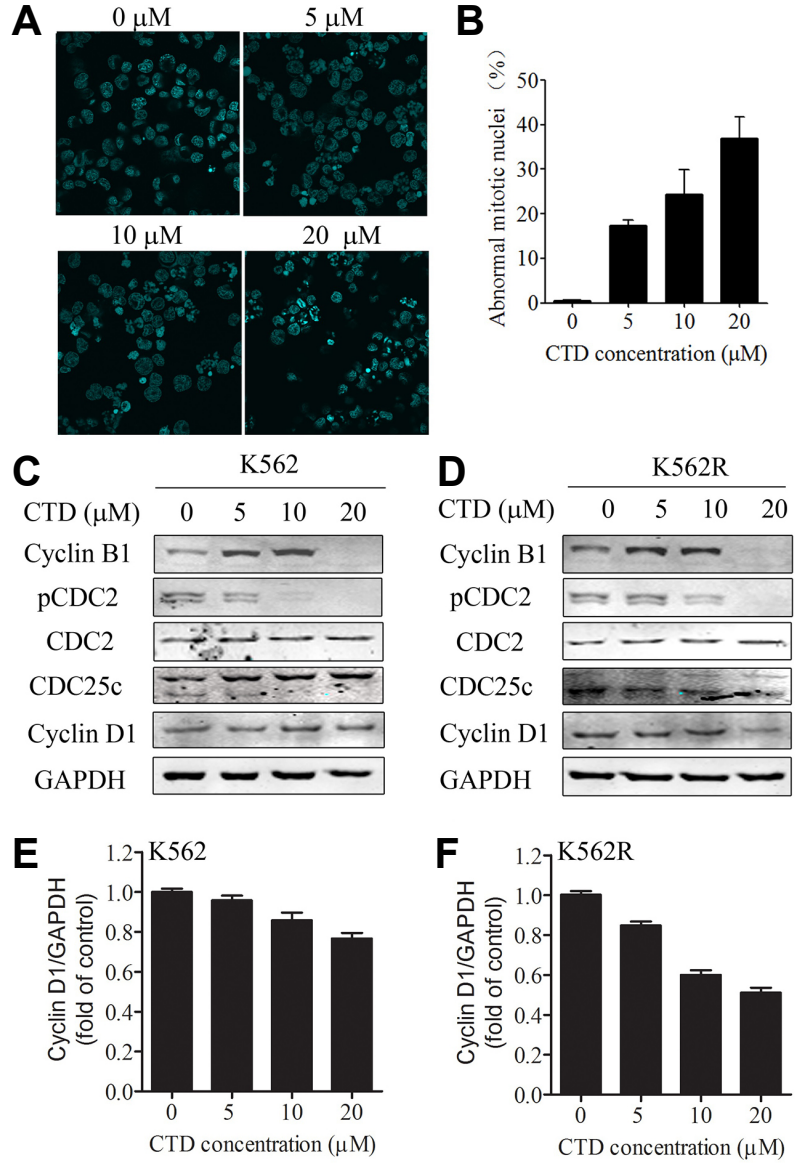

Fig. 3. CTD activated cyclin B1/ Cdc2 signaling pathway. (A) Representative Hoechst 33258 staining of K562 cells treated with indicated concentrations of CTD for $24 \mathrm{~h}$. (B) Quantification of abnormal mitotic nuclei treated with CTD. (C) K562 cells were treated with CTD $(0-20 \mu \mathrm{M})$ for $24 \mathrm{~h}$, and the expression of cyclin B1, Cdc2, pCdc2, Cdc25c, and cyclin D1 proteins were assessed by Western blot analyses and normalized relative to the expression of GAPDH. (D) K562R cells were treated with CTD $(0-20 \mu \mathrm{M})$ for $24 \mathrm{~h}$, and the expression of cyclin B1, Cdc2, pCdc2, Cdc25c, and cyclin D1 proteins were assessed by Western blot analyses and normalized relative to the expression of GAPDH. (E) Quantification of cyclinD1 expression in CTD-treated K562 cells. (F) Quantification of cyclinD1 expression in CTD-treated K562R cells.

\section{CTD induced DNA damage in CML cells}

As DNA damage was shown to be linked with cell cycle arrest, experiments to detect the occurrence of DNA damage were conducted. As shown in Fig. $4 \mathrm{~A}$, an increase in $\mathrm{YH} 2 \mathrm{AX}$ foci was observed in K562 cells treated with $20 \mu \mathrm{M}$ CTD for $24 \mathrm{~h}$. Subsequently, we assessed the expression levels of $\mathrm{yH} 2 \mathrm{AX}$ by immunoblotting. As shown in Fig. 4B, CTD triggered an increase in $\mathrm{YH} 2 \mathrm{AX}$ in a dose-dependent manner in both $\mathrm{K} 562$ and K562R cells. K562 and K562R cells were treated with 10 $\mu \mathrm{M}$ CTD for 0-24 $\mathrm{h}$, the expression of $\mathrm{yH} 2 \mathrm{AX}$ increased in a time-dependent manner (Fig. 4C).

DNA damage signaling pathway and mitotic arrest

Previous studies have demonstrated that several compounds, 
A

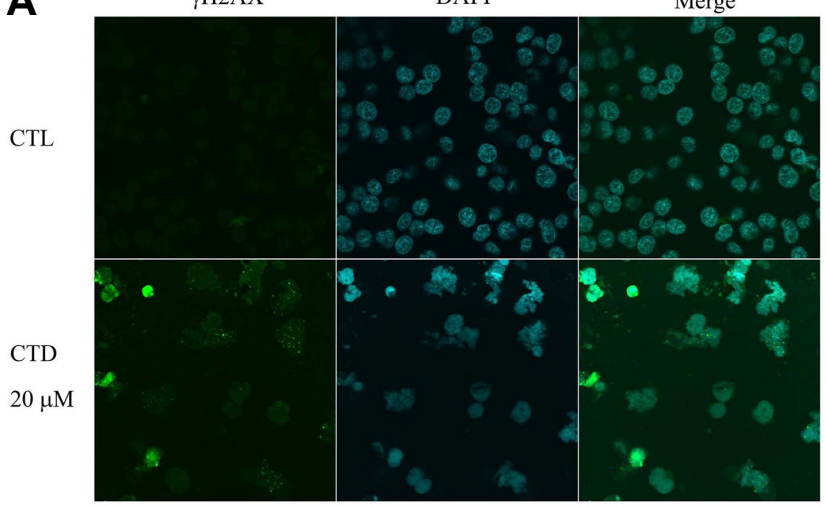

B

C

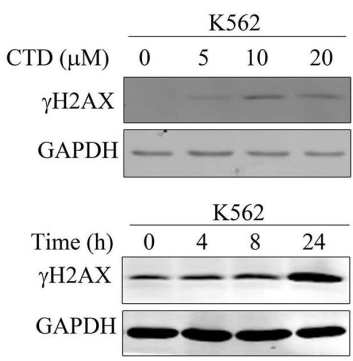

K562R

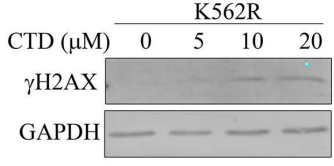

K562R

\begin{tabular}{lllll}
\cline { 2 - 4 } Time (h) & 0 & 4 & 8 & 24 \\
\hline
\end{tabular}

$\gamma \mathrm{H} 2 \mathrm{AX} \longrightarrow \div$

GAPDH
Fig. 4. CTD induced DNA damage in CML cells. (A) K562 cells were incubated with $20 \mu \mathrm{M}$ CTD for $24 \mathrm{~h}$ and stained with antibody against $\mathrm{YH} 2 \mathrm{AX}$ (green). DNA was stained with DAPI (blue). (B) K562 and K562R cells were treated with different concentrations of CTD for $24 \mathrm{~h}$, and the expression of $\mathrm{yH} 2 \mathrm{AX}$ was assessed by western blotting, and normalized relative to the expression of GAPDH. (C) K562 and K562R cells were treated with $10 \mu \mathrm{M}$ CTD for different times, and the expression of $\mathrm{YH} 2 \mathrm{AX}$ was assessed by Western blotting and normalized relative to the expression of GAPDH.
A

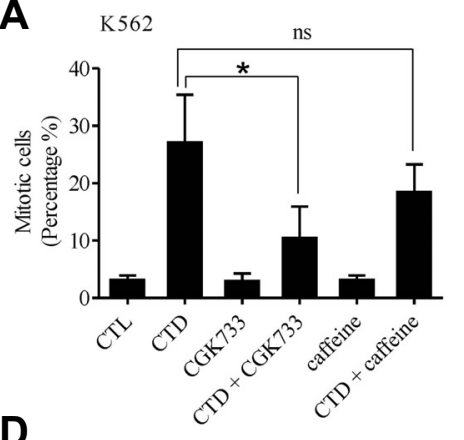

D

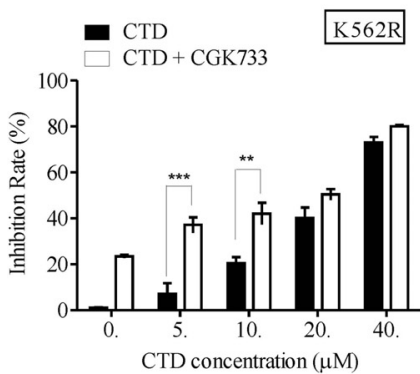

B

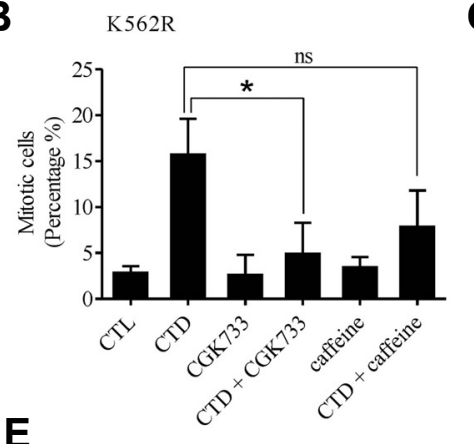

E

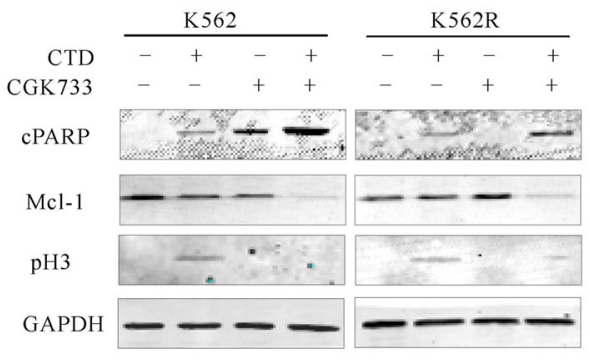

C

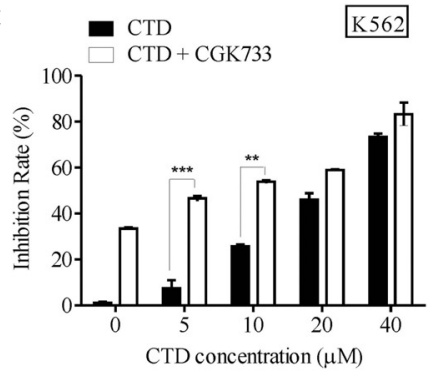

Fig. 5. DNA damage signaling pathway regulated CTD-mediated mitotic arrest. (A) K562 cells were pre-incubated for $1 \mathrm{~h}$ with either CGK733 $(5 \mu \mathrm{M})$ or caffeine $(2.5 \mathrm{mM})$, and then treated with CTD $(10 \mu \mathrm{M})$ for $24 \mathrm{~h}$. Cells in mitotic arrest were analyzed by flow cytometry. The asterisk $\left(^{*}\right)$ indicates a significant difference $(p<0.05)$ between CTD and CTD + CGK733 treated K562 cells. (B) K562R cells were pre-incubated for $1 \mathrm{~h}$ with either CGK733 $(5 \mu \mathrm{M})$ or caffeine $(2.5 \mathrm{mM})$, then treated with CTD $(10 \mu \mathrm{M})$ for $24 \mathrm{~h}$, and mitotic arrest was analyzed by flow cytometry. The asterisk $\left(^{*}\right)$ indicates a significant difference $(p<0.05)$ between CTD and CTD + CGK733 treated K562R cells. (C) K562 cells were pre-incubated for $1 \mathrm{~h}$ with CGK733 $(5 \mu \mathrm{M})$, then treated with indicated concentrations of CTD for $24 \mathrm{~h}$, and cell viability was evaluated by CCK-8 assay. The asterisks $\left.{ }^{* *},{ }^{* \star *}\right)$ indicate a significant difference $(p<0.01, p<$ 0.001) between CTD and CTD + CGK733 treated K562 cells. (D) K562R cells were pre-incubated for $1 \mathrm{~h}$ with CGK733 (5 $\mu \mathrm{M})$, then treated with indicated concentrations of CTD for $24 \mathrm{~h}$, and cell viability was evaluated by CCK-8 assay. The asterisks $\left({ }^{\star \star},{ }^{\star \star \star}\right)$ indicate a significant difference $(p<0.01, p<0.001)$ between CTD and CTD + CGK733 treated K562R cells.(E) K562 and K562R cells were pre-treated for $1 \mathrm{~h}$ with CGK733 $(5 \mu \mathrm{M})$, then treated with CTD $(10 \mu \mathrm{M})$ for $24 \mathrm{~h}$, and protein levels of cleaved PARP, Mcl-1, and phosphorylated $\mathrm{H} 3$ were determined by Western blotting. GAPDH served as a normal control. 


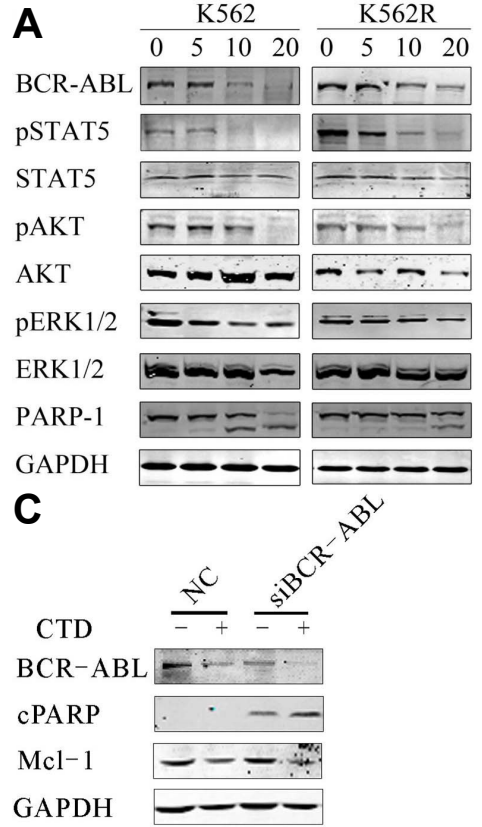

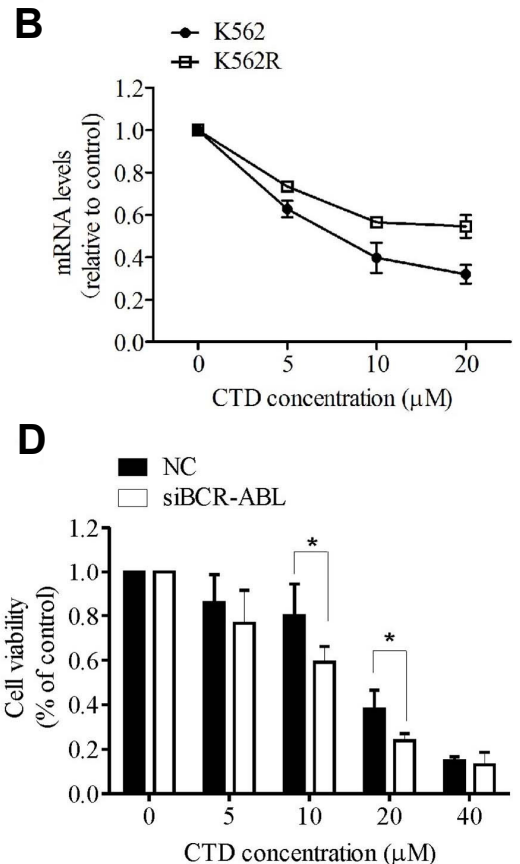

Fig. 6. CTD suppressed BCR-ABL signaling pathway. (A) K562 and K562R cells were treated with CTD at indicated concentrations for $24 \mathrm{~h}$. The protein expression of BCR-ABL and its downstream signaling molecules was analyzed by Western blotting. (B) K562 and $\mathrm{K} 562 \mathrm{R}$ cells were treated with CTD at indicated concentrations for $24 \mathrm{~h}$. The mRNA levels of BCR-ABL were measured by qRT-PCR. (C) K562 cells were transfected with scramble siRNA (negative control, $\mathrm{NC}$ ) or BCR-ABL specific siRNA. After $48 \mathrm{~h}$, the transfected cells were treated with $10 \mu \mathrm{M}$ CTD for additional $24 \mathrm{~h}$ and the expression of BCR-ABL, cleaved PARP and Mcl-1 proteins was analyzed by Western blotting. GAPDH served as a normal control. (D) Growth of NC or BCR-ABL specific siRNA-transfected K562 cells treated with indicated concentrations of CTD for $24 \mathrm{~h}$ was evaluated by CCK-8 assay. The asterisk $\left(^{*}\right)$ indicates a significant difference $(p<0.05)$ between CTD treated NC and siBCR-ABL K562 cells. such as curcumin (Blakemore et al., 2013) and diallyl trisulfide (Xiao et al., 2009), induced mitotic arrest involving DNA damage, and that inhibiting DNA damage signaling pathway abrogated the mitotic arrest. Therefore, we were interested in studying whether DNA damage signaling pathway was associated with CTD-induced mitotic arrest and cell death. K562 or K562R cells were treated with CTD in the presence or absence of caffeine (a pan-inhibitor of DNA damage network signaling kinases, ATM, ATR, DNA-PK and Chk1), and cell cycle status was evaluated. As shown in Figs. 5A and 5B, caffeine reduced CTD-induced mitotic arrest, although the reduction was not statistically significant. One explanation may be that we used caffeine at the concentration of $2.5 \mathrm{mM}$, which is much lower than the $10 \mathrm{mM}$ used in previous studies (Blakemore et al., 2013). In our experiment, $10 \mathrm{mM}$ caffeine caused significant cell death in K562 cells, and hence, we reduced the concentration to $2.5 \mathrm{mM}$. To further verify the role of DNA damage signaling kinases in CTD-mediated mitotic arrest, K562 or K562R cells were treated with CTD in the presence or absence of ATM/ATR inhibitor, CGK733, and the cell cycle arrest, cell viability and the levels of cleaved PARP, $\mathrm{Mcl}-1$, and $\mathrm{pH} 3$ proteins were evaluated. We found that CGK733 significantly abrogated the CTD-mediated mitotic arrest (Figs. 5A and 5B), but enhanced cell death (Figs. 5C and 5D) as evidenced by increased level of cleaved PARP and decreased level of $\mathrm{Mcl}-1$ (Fig. 5E). These results confirmed that inhibition of CTD-induced mitotic arrest leads to greater cell death in K562 and K562R cells.

\section{CTD depleted BCR-ABL and its downstream signal pathway}

The presence of oncoprotein $\mathrm{BCR}-\mathrm{ABL}$ is the main characteristic of CML (Deininger et al., 2000; Pane et al., 1996). Increased expression of $B C R-A B L$ and mutations in the tyrosine kinase domain of BCR-ABL are the major mechanisms underlying imatinib resistance (Bixby and Talpaz, 2010). Therefore, we examined whether CTD has any impact on BCR-ABL. CTD treatment significantly decreased $B C R-A B L$ in a dosedependent manner (Fig. 6A). CTD also inhibited the expression of the downstream target proteins of BCR-ABL. The phosphorylation of STAT5, AKT, and ERK1/2 was significantly decreased, whereas there was no dramatic change in the amount of total proteins. The downstream events of decreased BCR$A B L$ also included PARP cleavage. To study the mechanism of CTD-induced reduction of BCR-ABL protein, we measured the expression of BCR-ABL at the transcription level. K562 and K562R cells were treated with CTD or vehicle for $16 \mathrm{~h}$, and then RNA was extracted. qRT-PCR data revealed a clear decrease in BCR-ABL mRNA levels in both cell lines (Fig. 6B), suggesting that CTD-induced suppression of BCR-ABL transcription may be responsible for the decreased BCR-ABL protein levels.

To further investigate whether BCR-ABL is the cause of cellkilling effect of CTD, we knocked down BCR-ABL in K562 cells. We first examined the CTD-mediated reduction of BCR-ABL protein in the cells transfected with scramble siRNA and siBCR$A B L$. The results showed that the degree of BCR-ABL protein reduction in siBCR-ABL-transfected cells is more notable than it is in scramble siRNA-transfected cells (Fig. 6C). In addition, CTD triggered dramatic increase in cleaved PARP level and decrease in the level of Mcl-1 in BCR-ABL knockdown cells. Subsequently, we compared the cell-killing effect of CTD on scramble siRNA and siBCR-ABL-transfected cells. Since the cell viability was altered by the knockdown of BCR-ABL, we designated the growth rate of CTD-untreated, siRNA negative control cells as $100 \%$ for comparing the cell-killing effect of CTD on the the siRNA negative control and siBCR-ABL K562 groups. The results indicated that BCR-ABL knockdown cells were more sensitive to the cytotoxic effects of CTD compared to negative control cells (Fig. 6D). These findings suggested that the cell-killing effects of CTD are at least in part dependent on BCR-ABL protein downregulation. 


\section{DISCUSSION}

CTD is an active constituent of blister beetles of the genus Mylabris, which is a folk medicine used for over 2000 years in China (Wang, 1989). Recently, CTD and its derivatives have shown a strong anti-tumor activity against the cells of various cancers (Huang et al., 2011; Kuo et al., 2011; Li et al., 2010; 2011a; 2011b; Shou et al., 2013; Su et al., 2015; Zhang et al., 2014). It has been shown that CTD induced G2/M cell cycle arrest and apoptosis in human pancreatic cancer cells, melanoma cells, and colorectal cancer cells (Hsiao et al., 2014; Huang et al., 2011; Li et al., 2010). CTD also inhibited cell growth and promoted mitochondria-dependent apoptosis in cancer cells (Hsia et al., 2014; Kuo et al., 2010; Tian et al., 2015). CTD exerted its anti-tumor effect through inducing DNA damage and inhibiting DNA repair associated proteins in human lung cancer cells, $\mathrm{NCl}-\mathrm{H} 460$, and human bladder cancer cells (Hsia et al., 2015; Kuo et al., 2015). Furthermore, CTD suppressed the migration and invasion of cancer cells by decreasing $\beta$-catenin protein and suppressing matrix metalloproteinase-2/9 signaling (Huang et al., 2013; Wu et al., 2014). In this study, we investigated the anticancer activity of CTD on imatinib-sensitive and imatinib-resistant CML cell lines. CTD caused substantial growth inhibition and cell death in K562 and K562R cells, displaying little toxicity towards normal blood cells (Fig. 1). Further study of this activity revealed that CTD triggered cell cycle arrest at mitotic phase and induced DNA damage in CML cells (Figs. 2 and 4). Notably, CTD downregulated BCR-ABL and inhibited its downstream signaling pathway (Fig. $6 A)$. Knocking down BCR-ABL sensitized the K562 cells to CTD-mediated cell death (Fig. 6D). Our results may expand the potential use of CTD in overcoming imatinib resistance in CML.

An earlier study showed that CTD disrupted the metaphase alignment of chromosomes and induces a prolonged mitotic arrest in A549 cells (Bonness et al., 2006). Remarkably, the dose of CTD that induced mitotic arrest did not result in apoptosis in A549 cells, because these cells can progress from metaphase into anaphase after a 10 to 15-hour delay. In this study, we showed that CTD causes accumulation of K562 and K562R cells in mitotic phase, as indicated by the morphologic changes and increased expression of $\mathrm{pH} 3$ (S10) (Fig. 2). Hoechst 33258 staining indicated chromosomal condensation (Fig. 3A). The ATM/ATR inhibitor, CGK733, abrogated CTD-mediated mitotic arrest but promoted cell death in CML cells (Fig. 5). Although we are not sure whether CTD-treated CML cells can later enter anaphase, CTD-induced mitotic arrest may not result in cell death.

We found that CTD-induced $\mathrm{YH} 2 \mathrm{AX}$ accumulation and DNA damage signaling pathway regulate CTD-induced mitotic arrest in CML cells. As shown in Figs. $5 \mathrm{~A}$ and $5 \mathrm{~B}$, caffeine and CGK733 can reduce the degree of CTD-induced mitotic arrest. Indeed, DNA damage signaling pathway is closely associated with mitotic arrest (Blakemore et al., 2013; Xiao et al., 2009). In Caco-2 and HCT116 cells, curcumin disrupted the chromosomal alignment in mitotic cells and arrested the cells at the prophase/prometaphase stage of mitosis. Pretreatment with caffeine abrogated curcumin-induced mitotic arrest. The authors proposed that DNA damage might contribute to mitotic arrest caused by curcumin. The compound, diallyl trisulfide, also triggers Chk1-mediated mitotic arrest and drives apoptotic DNA fragmentation in LNCaP and HCT116 cells. These reports, together with our work, suggest that DNA damage signaling pathway is closely associated with mitotic arrest.

Imatinib resistance is becoming a great challenge in the treatment of CML patients. Although new generations of TKIs have been developed to overcome the resistance to imatinib, most of them are extremely expensive and their effects need further clinical investigations (Apperley, 2015). A novel strategy to overcome imatinib resistance could possibly be downregulating the addiction oncogene, BCR-ABL, at mRNA level or promoting the degradation of BCR-ABL protein (Chen et al., 2014; Lu et al., 2010; Shi et al., 2014). In this study, we reported that CTD decreased BCR-ABL expression and contributed to cell death in CML cells (Fig. 6). BCR-ABL is a constitutively active tyrosine kinase that phosphorylates several substrates and activates multiple signal transduction pathways. CTD treatment results in dose-dependent downregulation of BCR-ABL and its downstream signaling pathways (Fig. 6A). Further study revealed that CTD depleted BCR-ABL at transcriptional level (Fig. $6 B)$. We also showed that BCR-ABL knockdown makes the cells sensitive to CTD (Figs. $6 \mathrm{C}$ and $6 \mathrm{D}$ ). Even though CTD might be affecting multiple molecules, depleting BCR-ABL is at least one of the major factors that induce cell death in CML cells.

Previous reports have demonstrated that the phosphatase activity of PP2A is suppressed by the BCR-ABL enhanced expression of PP2A inhibitor SET, and reactivating PP2A can kill both TKI-sensitive and TKI-resistant CML cells (Neviani, 2005). It is known that CTD is a selective PP2A inhibitor, which seems contradictory to previous reports. We reasoned that CTD-induced downregulation of BCR-ABL mRNA is independent of its inhibitory effects on PP2A.

Recently, it has been demonstrated that CTD (0.2 and 1.0 $\mathrm{mg} / \mathrm{kg}$ ) treatment led to a reduction of tumor size in A431 xenograft mouse model (Li et al., 2016). Han et al. (2013) also reported that CTD treatment $(0.25,0.5$, and $1.0 \mathrm{mg} / \mathrm{kg})$ caused significant regression of $S_{180}$ cell xenograft tumors in ICR male mice. In this study, treatment of CTD induced cell death in K562 and K562R cells at the concentration of 5-80 $\mu \mathrm{M}$ (Figs. 1D and 1E). In addition, the CTD-mediated BCR-ABL depletion effect was observed at 10 and $20 \mu \mathrm{M}$ concentrations after $24 \mathrm{~h}$ treatment (Fig. 6A), suggesting that CTD has an anticancer effect in CML xenograft murine models. Therefore, more investigations on the anticancer effects of CTD on CML murine models are needed in future.

Note: Supplementary information is available on the Molecules and Cells website (www.molcells.org).

\section{ACKNOWLEDGMENTS}

We thank Prof. Guangbiao Zhou (Institute of Zoology, Chinese Academy of Sciences, China) for kindly providing the imatinibresistant cell line K562R. This work was financially supported by National Natural Science Foundation of China (No. 81202576, 81503311), Natural Science Foundation of Jiangsu Province Grant (BK20131038).

\section{REFERENCES}

Apperley, J.F. (2015). Chronic myeloid leukaemia. Lancet 385, 1447-1459.

Baselga, J., and Arribas, J. (2004). Treating cancer's kinase 'addiction'. Nat. Med. 10, 786-787.

Bixby, D., and Talpaz, M. (2010). Seeking the causes and solutions to imatinib-resistance in chronic myeloid leukemia. Leukemia 25, 7-22.

Blakemore, L.M., Boes, C., Cordell, R., and Manson, M.M. (2013). Curcumin-induced mitotic arrest is characterized by spindle abnormalities, defects in chromosomal congression and DNA damage. Carcinogenesis 34, 351-360.

Bonness, K., Aragon, I.V., Rutland, B., Ofori-Acquah, S., Dean, N.M., and Honkanen, R.E. (2006). Cantharidin-induced mitotic arrest is associated with the formation of aberrant mitotic spindles and lagging chromosomes resulting, in part, from the 
suppression of PP2A alpha. Mol. Cancer Ther. 5, 2727-2736.

Chen, X., Shi, X., Zhao, C., Li, X., Lan, X., Liu, S., Huang, H., Liu, N., Liao, S., Zang, D., et al. (2014). Anti-rheumatic agent auranofin induced apoptosis in chronic myeloid leukemia cells resistant to imatinib through both Bcr/Abl-dependent and independent mechanisms. Oncotarget 5, 9118-9132.

Crosio, C., Fimia, G.M., Loury, R., Kimura, M., Okano, Y., Zhou, H., Sen, S., Allis, C.D., and Sassone-Corsi, P. (2002). Mitotic phosphorylation of histone $\mathrm{H} 3$ : spatio-temporal regulation by mammalian Aurora kinases. Mol. Cell Biol. 22, 874-885.

Danial, N.N., and Rothman, P. (2000). JAK-STAT signaling activated by Abl oncogenes. Oncogene 19, 2523-2531.

Deininger, M.W., Goldman, J.M., and Melo, J.V. (2000). The molecular biology of chronic myeloid leukemia. Blood 96, 3343-3356.

Deininger, M., Buchdunger, E., and Druker, B.J. (2005). The development of imatinib as a therapeutic agent for chronic myeloid leukemia. Blood 105, 2640-2653.

Gautier, J., Solomon, M.J., Booher, R.N., Bazan, J.F., and Kirschner, M.W. (1991). cdc25 is a specific tyrosine phosphatase that directly activates p34cdc2. Cell $67,197-211$.

Gong, F.R., Wu, M.Y., Shen, M., Zhi, Q., Xu, Z.K., Wang, R., Wang, W.J., Zong, Y., Li, Z.L., Wu, Y., et al. (2015). PP2A inhibitors arrest G2/M transition through JNK/Sp1-dependent downregulation of CDK1 and autophagy-dependent up-regulation of p21. Oncotarget 6, 18469-18483.

Gesbert, F., Sellers, W.R., Signoretti, S., Loda, M., and Griffin, J.D. (2000). BCR/ABL regulates expression of the cyclin-dependent kinase inhibitor p27Kip1 through the phosphatidylinositol 3Kinase/AKT pathway. J. Biol. Chem. 275, 39223-39230.

Han, W., Wang, S., Liang, R., Wang, L., Chen, M., Li H., and Wang, Y. (2013). Non-ionic surfactant vesicles simultaneously enhance antitumor activity and reduce the toxicity of cantharidin. Int. J. Nanomedicine 8, 2187-2196.

Hochhaus, A., O'Brien, S.G., Guilhot, F., Druker, B.J., Branford, S., Foroni, L., Goldman, J.M., Müller, M.C., Radich, J.P., Rudoltz, M., et al. (2009). Six-year follow-up of patients receiving imatinib for the first-line treatment of chronic myeloid leukemia. Leukemia 23 , 1054-1061.

Hsia, T.C., Yu, C.C., Hsu, S.C., Tang, N.Y., Lu, H.F., Huang, Y.P., Wu, S.H., Lin, J.G., and Chung, J.G. (2014). Cantharidin induces apoptosis of $\mathrm{H} 460$ human lung cancer cells through mitochondria-dependent pathways. Int. J. Oncol. 45, 245-254.

Hsia, T.C., Lin, J.H., Hsu, S.C., Tang, N.Y., Lu, H.F., Wu, S.H., Lin, J.G., and Chung, J.G. (2015). Cantharidin induces DNA damage and inhibits DNA repair-associated protein levels in $\mathrm{NCl}-\mathrm{H} 460$ human lung cancer cells. Environ. Toxicol. 30, 1135-1143.

Hsiao, Y.P., Tsai, C.H., Wu, P.P., Hsu, S.C., Liu, H.C., Huang, Y.P., Yang, J.H., and Chung, J.G. (2014). Cantharidin induces G2/M phase arrest by inhibition of Cdc25c and Cyclin A and triggers apoptosis through reactive oxygen species and the mitochondriadependent pathways of A375.S2 human melanoma cells. Int. J. Oncol. 45, 2393-2402.

Huang, W.W., Ko, S.W., Tsai, H.Y., Chung, J.G., Chiang, J.H., Chen, K.T., Chen, Y.C., Chen, H.Y., Chen, Y.F., and Yang, J.S. (2011). Cantharidin induces G2/M phase arrest and apoptosis in human colorectal cancer colo 205 cells through inhibition of CDK1 activity and caspase-dependent signaling pathways. Int. J. Oncol. 38, 1067-1073.

Huang, Y.P., Ni, C.H., Lu, C.C., Chiang, J.H., Yang, J.S., Ko, Y.C., Lin, J.P., Kuo, J.H., Chang, S.J., and Chung, J.G. (2013). Suppressions of migration and invasion by cantharidin in TSGH8301 human bladder carcinoma cells through the inhibitions of matrix metalloproteinase-2/-9 signaling. Evid. Based Complement Alternat. Med. eCAM 2013, 190281.

Kuo, J.H., Chu, Y.L., Yang, J.S., Lin, J.P., Lai, K.C., Kuo, H.M., Hsia, T.C., and Chung, J.G. (2010). Cantharidin induces apoptosis in human bladder cancer TSGH 8301 cells through mitochondriadependent signal pathways. Int. J. Oncol. 37, 1243-1250.

Kuo, J.H., Shih, T.Y., Lin, J.P., Lai, K.C., Lin, M.L., Yang, M.D., and Chung, J.G. (2015). Cantharidin induces DNA damage and inhibits DNA repair-associated protein expressions in TSGH8301 human bladder cancer cell. Anticancer Res. 35, 795-804.

Li, W., Chen, Z., Gong, F.R., Zong, Y., Chen, K., Li, D.M., Yin, H., Duan, W.M., Miao, Y., Tao, M., et al. (2011a). Growth of the pancreatic cancer cell line PANC-1 is inhibited by protein phosphatase $2 \mathrm{~A}$ inhibitors through over activation of the c-Jun N- terminal kinase pathway. Eur. J. Cancer 47, 2654-2664.

Li, W., Chen, Z., Zong, Y., Gong, F.R., Zhu, Y., Zhu, Y., Lv, J., Zhang, J., Xie, L., Sun, Y., et al. (2011b). PP2A inhibitors induce apoptosis in pancreatic cancer cell line PANC-1 through persistent phosphorylation of IKKa and sustained activation of the NF-kB pathway. Cancer Lett. 304, 117-127.

Li, W., Xie, L., Chen, Z., Zhu, Y., Sun, Y., Miao, Y., Xu, Z., and Han, $X$. (2010). Cantharidin, a potent and selective PP2A inhibitor, induces an oxidative stress-independent growth inhibition of pancreatic cancer cells through G2/M cell-cycle arrest and apoptosis. Cancer Sci. 101, 1226-1233.

Li, C.C., Yu, F.S., Fan, M.J., Chen, Y.Y., Lien, J.C., Chou, Y.C., Lu, H.F., Tang, N.Y., Peng, S.F., Huang, W.W., et al. (2016). Anticancer effects of cantharidin in A431 human skin cancer (Epidermoid carcinoma) cells in vitro and in vivo. Environ. Toxicol. 2016 doi: 10.1002/tox.22273 (in press).

Lu, Z., Jin, Y., Qiu, L., Lai, Y., and Pan, J. (2010). Celastrol, a novel HSP90 inhibitor, depletes Bcr-Abl and induces apoptosis in imatinib-resistant chronic myelogenous leukemia cells harboring T315I mutation. Cancer Lett. 290, 182-191.

Lugo, T.G., Pendergast, A.M., Muller, A.J., and Witte, O.N. (1990). Tyrosine kinase activity and transformation potency of bcr-abl oncogene products. Science 247, 1079-1082.

Neviani, P., Santhanam, R., Trotta, R., Notari, M., Blaser, B.W., Liu, S., Mao, H., Chang, J.S., Galietta, A., Uttam, A., et al. (2005). The tumor suppressor PP2A is functionally inactivated in blast crisis CML through the inhibitory activity of the BCR/ABLregulated SET protein. Cancer Cell 8, 355-336.

Norbury, C., Blow, J., and Nurse P. (1991). Regulatory phosphorylation of the p34cdc2 protein kinase in vertebrates. EMBO J. 10, 3321-3329.

Pane, F., Frigeri, F., Sindona, M., Luciano, L., Ferrara, F., Cimino, R., Meloni, G., Saglio, G., Salvatore, F., and Rotoli, B. (1996). Neutrophilic-chronic myeloid leukemia: a distinct disease with a specific molecular marker (BCR/ABL with $\mathrm{C} 3 / \mathrm{A} 2$ junction). Blood. 88, 2410-2414.

Sánchez, I., and Dynlacht, B.D. (2005). New insights into cyclins, CDKs, and cell cycle control. Semin. Cell Dev. Biol. 16, 311-321.

Shi, X., Chen, X., Li, X., Lan, X., Zhao, C., Liu, S., Huang, H., Liu, N., Liao, S., Song, W., et al. (2014). Gambogic acid induces apoptosis in imatinib-resistant chronic myeloid leukemia cells via inducing proteasome inhibition and caspase-dependent Bcr-Abl downregulation. Clin. Cancer Res. 20,151-163.

Shou, L.M., Zhang, Q.Y., Li, W., Xie, X., Chen, K., Lian, L., Li, Z.Y., Gong, F.R., Dai, K.S., Mao, Y.X., et al. (2013). Cantharidin and norcantharidin inhibit the ability of MCF-7 cells to adhere to platelets via protein kinase $C$ pathway-dependent downregulation of a2 integrin. Oncol. Rep. 30, 1059-1066.

Su, C.C., Liu, S.H., Lee, K.I., Huang, K.T., Lu, T.H., Fang, K.M., Wu, C.C., Yen, C.C., Lai, C.H., Su, Y.C., et al. (2015) Cantharidin induces apoptosis through the Calcium/PKC-regulated endoplasmic reticulum stress pathway in human bladder cancer cells. Am. J. Chin. Med. 43, 581-600.

Tian, X., Zeng, G., Li, X., Wu, Z., and Wang, L. (2015). Cantharidin inhibits cell proliferation and promotes apoptosis in tongue squamous cell carcinoma through suppression of miR-214 and regulation of p53 and Bcl-2/Bax. Oncol. Rep. 33, 3061-3068.

Wang, G.S. (1989). Medical uses of mylabris in ancient China and recent studies. J. Ethnopharmacol. 26, 147-162.

Wolanin, K., Magalska, A., Mosieniak, G., Klinger, R., McKenna, S., Vejda, S., Sikora, E., and Piwocka, K. (2006). Curcumin affects components of the chromosomal passenger complex and induces mitotic catastrophe in apoptosis-resistant Bcr-Ablexpressing cells. Mol. Cancer Res. 4, 457-469.

Wu, M.Y., Xie, X., Xu, Z.K., Xie, L., Chen, Z., Shou, L.M., Gong, F.R., Xie, Y.F., Li, W., and Tao, M. (2014). PP2A inhibitors suppress migration and growth of PANC-1 pancreatic cancer cells through inhibition on the $W n t / \beta$-catenin pathway by phosphorylation and degradation of $\beta$-catenin. Oncol. Rep. 32, 513-522.

Xiao D., Zeng Y., and Singh S.V. (2009). Diallyl trisulfide-induced apoptosis in human cancer cells is linked to checkpoint kinase 1mediated mitotic arrest. Mol. Carcinog. 48, 1018-1029.

Zhang, W., Ma, Y.Z., Song, L., Wang, C.H., Qi, T.G., and Shao, G.R. (2014). Effect of cantharidins in chemotherapy for hepatoma: a retrospective cohort study. Am. J. Chin. Med. 42, 561-567. 